Case Report

\title{
BK Virus Encephalitis in HIV-Infected Patients: Case Report and Review
}

\author{
Luciana Antoniolli, Rafael Borges, and Luciano Z. Goldani \\ Infectious Disease Section, Hospital de Clínicas de Porto Alegre, Universidade Federal do Rio Grande do Sul, Ramiro Barcelos 2350, \\ 90640-000 Porto Alegre, RS, Brazil \\ Correspondence should be addressed to Luciano Z. Goldani; lgoldani@ufrgs.br
}

Received 1 August 2016; Revised 26 December 2016; Accepted 23 January 2017; Published 23 February 2017

Academic Editor: Walter Zidek

Copyright (C) 2017 Luciana Antoniolli et al. This is an open access article distributed under the Creative Commons Attribution License, which permits unrestricted use, distribution, and reproduction in any medium, provided the original work is properly cited.

\begin{abstract}
Encephalitis and meningitis due to BKPyV are unusual and emerging condition. Only a few cases of BKPyV encephalitis have been reported in hematopoietic stem cell transplant recipients, with the majority of cases presenting with concurrent hemorrhagic cystitis and HIV-infected patients. The authors report two HIV-infected patients with the diagnosis of BKPyV encephalitis and discuss the main clinical, diagnostic, and therapeutic aspects of this infection in patients with AIDS. Physicians should be aware to recognize the main clinical features and diagnose BKPyV central nervous infection in the setting of AIDS.
\end{abstract}

\section{Introduction}

BK virus (BKPyV) JC virus (JCPyV) and simian 40 (SV40) are members of the Polyomaviridae family [1]. BKPYV infection affects $60-90 \%$ of the general population and occurs during childhood via the respiratory tract and is usually asymptomatic [2]. The virus remains latent after infection, particularly in the kidneys and other tissues including the brain $[1,2]$. Viral reactivation occurring especially in immunocompromised patients may manifest as hemorrhagic cystitis, ureteral stenosis, tubulointerstitial nephritis, retinitis, encephalitis, and pneumonia [3]. Encephalitis and meningitis due to $\mathrm{BKPyV}$ are unusual and emerging condition with most cases being reported in HIV-infected patients or after transplantation $[4,5]$. The authors report two HIV-infected patients with the diagnosis of $\mathrm{BKPyV}$ encephalitis and discuss the main clinical, diagnostic, and therapeutic aspects of this infection in patients with AIDS.

\section{Case Reports}

2.1. Case 1. A 52-year-old man was admitted to the emergency room because of a headache, fever $\left(39^{\circ} \mathrm{C}\right)$, alternating with hypothermia, confusion, and weakness in the left leg.
The patient also reported diarrhea, loss of appetite, and weight loss of 12 kilograms in the past three months. He had been diagnosed with HIV infection a few days before his admission. He denied previous diseases, using continuous medication, and previous hospital admissions or surgeries.

At the physical examination, the patient presented a regular status with confusion, isochoric and photoreactive pupils, and no signs of meningeal irritation, but a loss of force in the left leg. Computed tomography (CT) scan showed an area of hypodensity in the white matter on the upper convexity of the right frontal lobe. A magnetic resonance image (MRI) showed a hypersignal in T2 on the upper and lower right frontal gyri, in the left occipital lobe, and lesions with ring enhancement in the deep upper left temporal sulcus (Figure 1). Laboratory exams showed a lymphocyte CD4 count of 94 cells $/ \mathrm{mm}^{3}$, and HIV viral load of 479,365 copies $\mathrm{ml}$. A reactive serological test for syphilis showed a titer $1: 8$, which was previously treated with penicillin G benzathine. A lumbar puncture was performed, and the cerebrospinal fluid (CSF) analysis showed 3 leucocytes $/ \mathrm{mm} 3$, proteins of $28 \mathrm{mg} / \mathrm{dL}$, and glucose of $39 \mathrm{mg} / \mathrm{dL}$. CSF cultures were negative for bacteria, mycobacteria, and fungi. PCR testing for $\mathrm{JCPyV}$ virus was negative. However, PCR testing for BKPyV was positive. Detection of BKPyV consisted of a semi-nested 


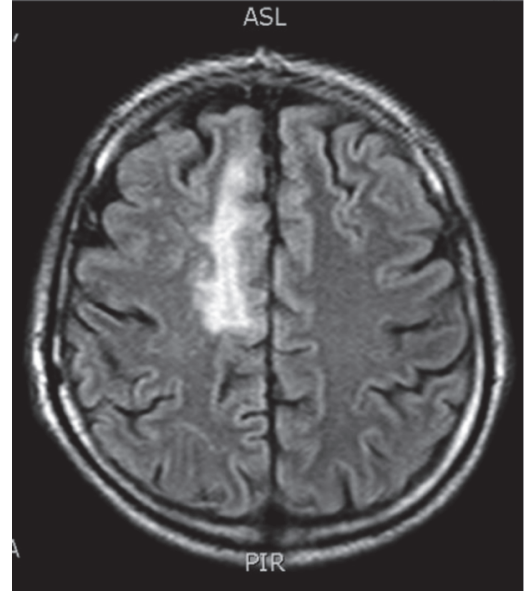

FIGURE 1: A magnetic resonance image showed a hyperintensity in $\mathrm{T} 2$ on the upper and lower right frontal gyri, in the left occipital lobe, and lesions with annular enhancement in the deep upper left temporal sulcus (case report 1).

PCR with two 20-base oligomer primers (PEP-1 and PEP-2) followed by second round PCR with 40-nucleotide sequence (BEP-1 and PEP-1), as previously described [6]. The length of the BKPyV targeted for amplification was 176 nucleotide pairs. At this same hospitalization, the patient was diagnosed with pulmonary tuberculosis, and a regime of rifampin, ethambutol, pyrazinamide, and isoniazid was provided. The patient was started on highly active antiretroviral therapy (HAART). However, he presented signs of sepsis of unknown origin twenty days after the admission and died three days later.

2.2. Case 2. A 45-year-old woman was admitted to this hospital because of headaches, epigastric pain, nausea, vomiting, asthenia, dyspnea, loss of appetite, and fever. She had been diagnosed with HIV infection one month before. The patient was taking sulfadiazine and pyrimethamine due to a presumptive diagnosis of neurotoxoplasmosis in a previous recent hospitalization. On the physical examination, the patient was lethargic, with pain at palpation. She presented a severe immunosuppression with a CD4-T lymphocyte count of 23 cells $/ \mathrm{mm}^{3}$ and a viral load of 167,580 copies $/ \mathrm{ml}$. Cerebral MRI showed mild volume loss with scattered FLAIR hyperintensities and asymmetric lesions without mass effect (Figure 2). The cerebrospinal fluid analysis showed 8 leucocytes $/ \mathrm{mm} 3$, protein $133 \mathrm{mg} / \mathrm{dL}$, and glucose $40 \mathrm{mg} / \mathrm{dl}$. Cultures for bacteria, mycobacteria, and fungi were negative. PCR testing for herpes simplex virus types 1 and 2, herpes zoster virus, Epstein-Barr virus, and JC virus was negative. However, the sample was positive for the presence of $\mathrm{BKPyV}$ and cytomegalovirus. She continued to receive empiric treatment for neurotoxoplasmosis. During the hospitalization, she presented impaired renal function, which was attributed to sulfadiazine. It was also considered the hypothesis of BK renal infection, reinforced by a positive PCR testing for BKPYV in the urine. The patient was started on HAART (zidovudine,

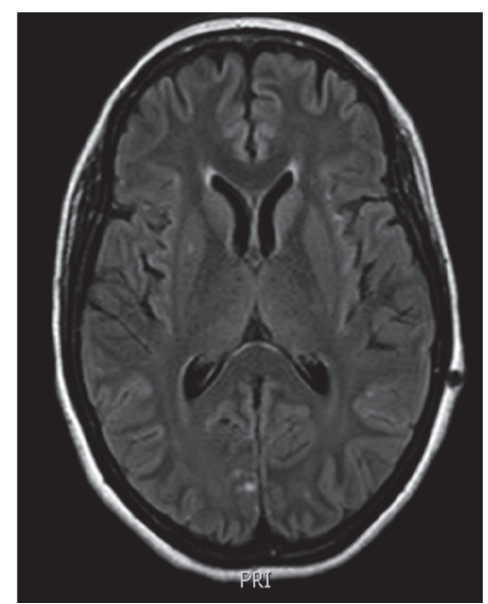

FIGURE 2: Cerebral MRI currently shows mild volume loss with scattered FLAIR hyperintensities and asymmetric lesions without mass effect (case report 2).

lamivudine, and efavirenz). She was discharged after 62 days of hospitalization with partial neurological improvement.

\section{Discussion}

$\mathrm{BKPyV}$ encephalitis has been reported in patients with depression of their immune function including patients with renal allograft: hematooncological diseases undergoing chemotherapy, bone marrow transplantation, long-term steroid therapy, and HIV [4-11]. However, BKPyV has been detected in asymptomatic apparently healthy and immunocompetent individuals, with self-limited clinical manifestations $[12,13]$. To date, since the first report of BKPyV associated neurological infection almost 20 years ago, only eight cases have been described in patients with AIDS (Table 1) [14-22]. As observed in our two patients, the most common symptom is a headache. Other signs of neurological impairment include seizures, progressive mental deterioration, dysarthria, hallucinations, visual disturbances, and in one case paraplegia. $\mathrm{BKPyV}$ retinitis has been described in AIDS patients [20]. Imaging of CNS infection by $\mathrm{BKPyV}$ is characterized by a preferential involvement of the periventricular and pial surfaces of the brain parenchyma. In fact, magnetic resonance imaging of the cases of $\mathrm{BKPyV}$ meningoencephalitis has shown areas of increased signal intensity of the periventricular white matter of brain while the cortex is generally spared $[5,9]$. Meningeal contrast enhancement along with increased meningeal thickness also occurs when infection spread to meningitis is present. Deep white matter of the cerebellum is generally spared while deep gray matter structures are compromised. As shown in Table 1, the diagnosis of BKPyV encephalitis has usually been established by PCR testing of the CSF in the majority of the patients and sometimes complemented or not by BKPyV PCR of the brain biopsy specimen. The lack of demonstration of the virus in the brain tissue sample has been frequently observed in the reported cases, either by immunohistochemistry or by in situ hybridization. If a patient shows neurological symptoms 


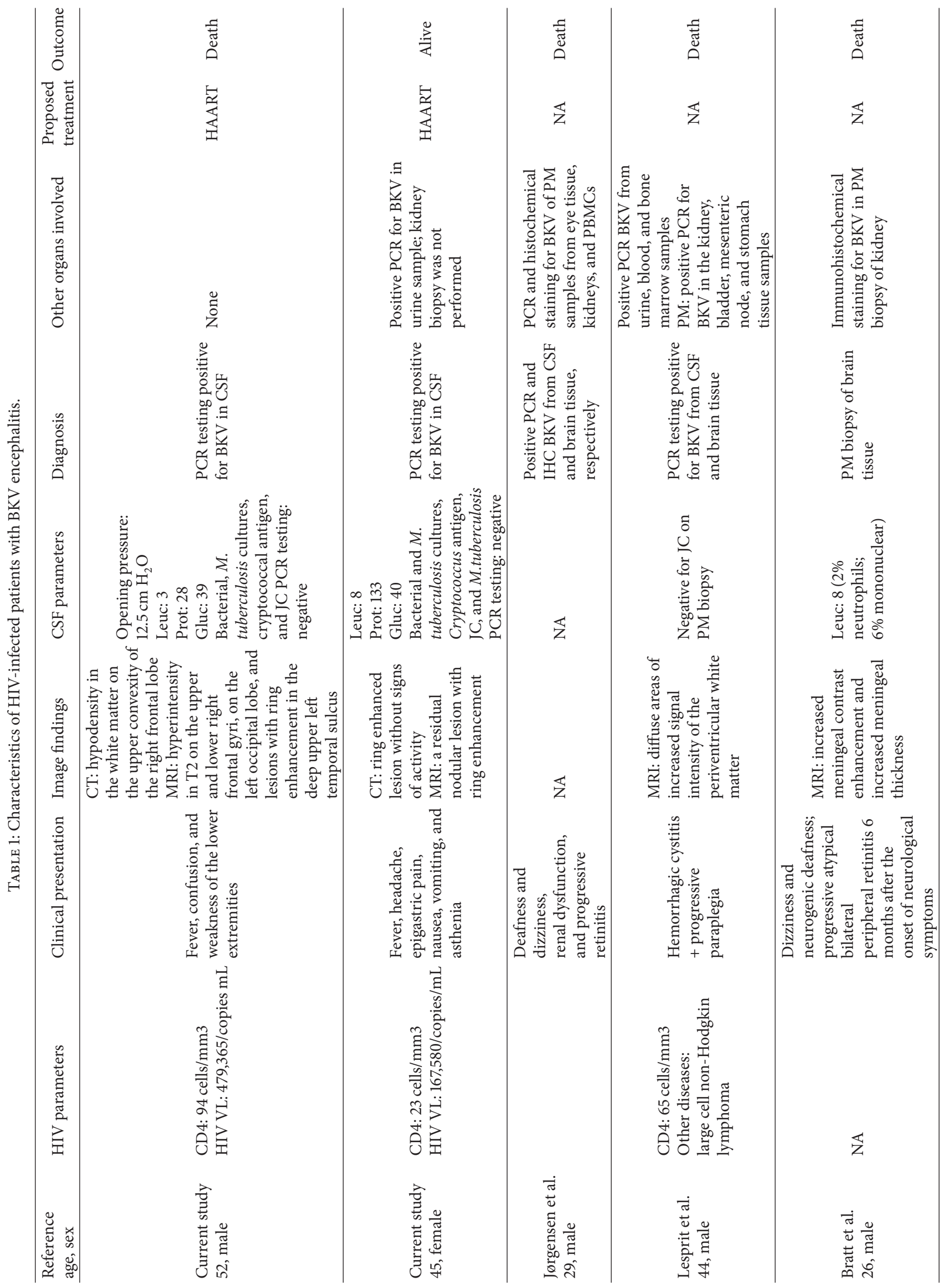




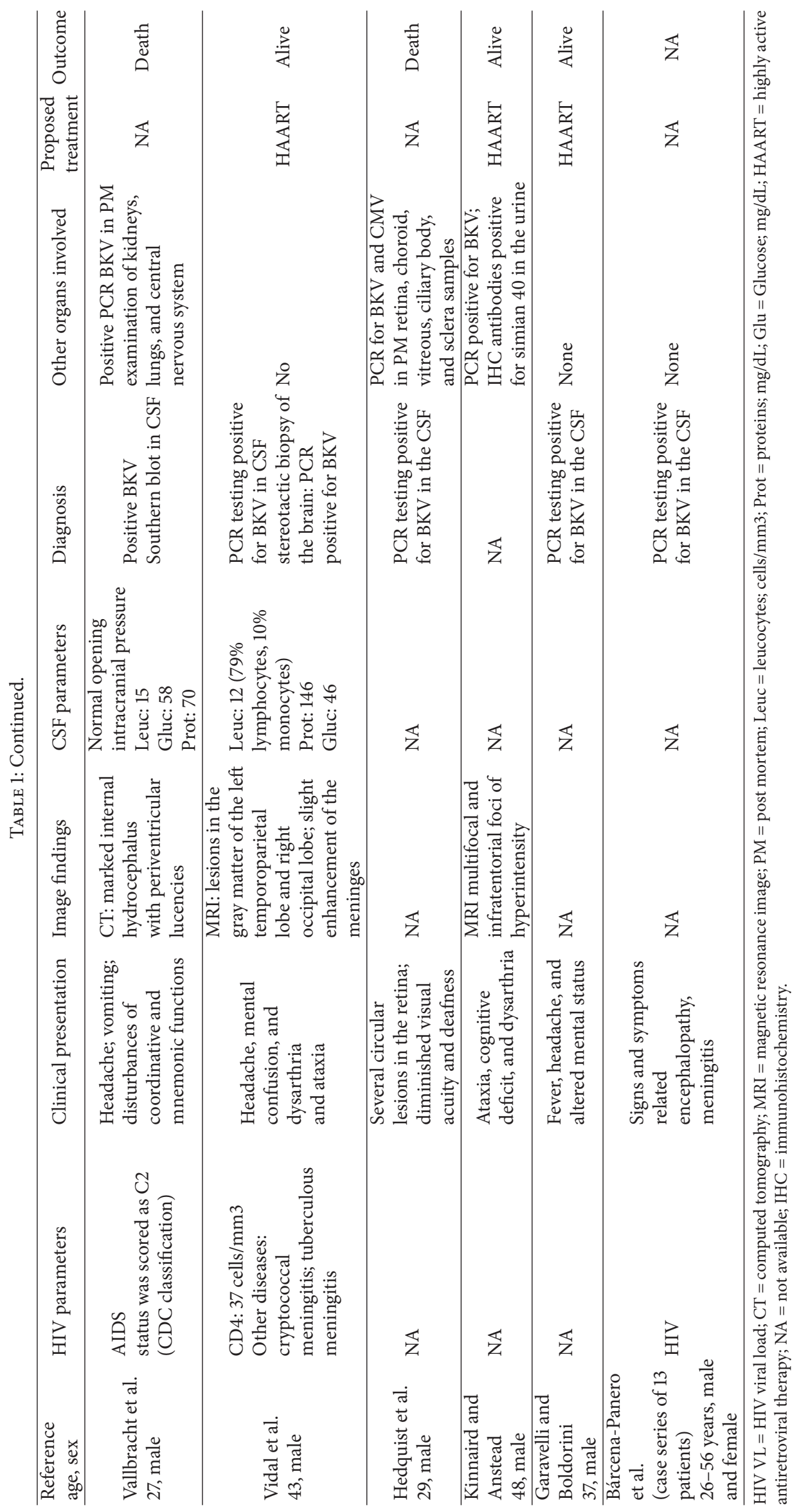


without a positive serology and PCR for other pathogens (namely, viral agents such as JCV, herpes simplex virus, and cytomegalovirus), while a positive PCR for BKPyV is present in the CSF/brain tissue, it strongly favors the diagnosis of a $\mathrm{BKPyV}$ meningoencephalitis.

There is no established treatment of BKPyV infection. Antiviral agents such as cidofovir, leflunomide, IVIG, and quinolones have been used in refractory cases of $\mathrm{BKPyV}$ nephropathy and cystitis [22-25]. However, the effectiveness of these agents is doubtful and some of them can cause severe side effects. However, in the few reported cases of $\mathrm{BKPyV}$ encephalitis, therapy has been restricted to the use of antiretroviral therapy. Consequently, overall mortality of the reported cases has been high, $70 \%$ of the reported cases in the literature. In summary, $\mathrm{BKPyV}$ meningoencephalitis is an emerging disease in HIV-infected patients. Physicians should be aware to recognize the main clinical features and diagnose $\mathrm{BKPyV}$ meningoencephalitis. Specific antiviral therapy is an urgent need for treatment of BKPyV infection.

\section{Competing Interests}

The authors declared that there are no competing interests regarding the publication of this paper.

\section{References}

[1] Polyomaviridae Study Group of the International Committee on Taxonomy of Viruses, S. Calvignac-Spencer, M. C. Feltkamp et al., "A taxonomy update for the family Polyomaviridae," Archives of Virology, vol. 161, no. 6, pp. 1739-1750, 2016.

[2] M. Jiang, J. R. Abend, S. F. Johnson, and M. J. Imperiale, “The role of polyomaviruses in human disease," Virology, vol. 384, no. 2, pp. 266-273, 2009.

[3] M. D. Reploeg, G. A. Storch, and D. B. Clifford, "BK virus: a clinical review," Clinical Infectious Diseases, vol. 33, no. 2, pp. 191-202, 2001.

[4] R. Boothpur and D. C. Brennan, "Human polyoma viruses and disease with emphasis on clinical BK and JC," Journal of Clinical Virology, vol. 47, no. 4, pp. 306-312, 2010.

[5] L. Vago, P. Cinque, E. Sala et al., "JCV-DNA and BKV-DNA in the CNS tissue and CSF of AIDS patients and normal subjects. Study of 41 cases and review of the literature," Journal of Acquired Immune Deficiency Syndromes and Human Retrovirology, vol. 12, no. 2, pp. 139-146, 1996.

[6] R. R. Arthur, S. Dagostin, and K. V. Shah, "Detection of BK virus and JC virus in urine and brain tissue by the polymerase chain reaction," Journal of Clinical Microbiology, vol. 27, no. 6, pp. 1174-1179, 1989.

[7] R. Lopes da Silva, I. Ferreira, G. Teixeira et al., "BK virus encephalitis with thrombotic microangiopathy in an allogeneic hematopoietic stem cell transplant recipient," Transplant Infectious Disease, vol. 13, no. 2, pp. 161-167, 2011.

[8] J. K. Hix, W. E. Braun, and C. M. Isada, "Delirium in a renal transplant recipient associated with $\mathrm{BK}$ virus in the cerebrospinal fluid," Transplantation, vol. 78, no. 9, pp. 1407-1408, 2004.

[9] L. Cabrejo, M. Diop, A. Blohorn-Sense, and B. Mihout, "Progressive BK virus associated multifocal leukoencephalopathy in an immunocompromised patient treated with corticosteroids," Revue Neurologique, vol. 161, no. 3, pp. 326-330, 2005.

[10] A. Ferrari, M. Luppi, R. Marasca et al., "BK virus infection and neurologic dysfunctions in a patient with lymphoma treated with chemotherapy and rituximab," European Journal of Haematology, vol. 81, no. 3, pp. 244-245, 2008.

[11] G. Behre, M. Becker, and M. Christopeit, "BK virus encephalitis in an allogeneic hematopoietic stem cell recipient," Bone Marrow Transplantation, vol. 42, no. 7, p. 499, 2008.

[12] R. Voltz, G. Jager, K. Seelos, L. Fuhry, and R. Hohlfeld, "BK virus encephalitis in an immunocompetent patient," Archives of Neurology, vol. 53, no. 1, pp. 101-103, 1996.

[13] C. Elsner and K. Dörries, "Evidence of human polyomavirus BK and JC infection in normal brain tissue," Virology, vol. 191, no. 1, pp. 72-80, 1992.

[14] A. Bárcena-Panero, J. E. Echevarría, M. Van Ghelue et al., "BK polyomavirus with archetypal and rearranged non-coding control regions is present in cerebrospinal fluids from patients with neurological complications," Journal of General Virology, vol. 93, no. 8, pp. 1780-1794, 2012.

[15] G. E. K. Jørgensen, A.-L. Hammarin, G. Bratt, M. Grandien, T. Flægstad, and J. I. Johnsen, "Identification of a unique BK virus variant in the CNS of a patient with AIDS," Journal of Medical Virology, vol. 70, no. 1, pp. 14-19, 2003.

[16] P. Lesprit, D. Chaline-Lehmann, F.-J. Authier, T. Ponnelle, F. Gray, and Y. Levy, "BK virus encephalitis in a patient with AIDS and lymphoma," AIDS, vol. 15, no. 9, pp. 1196-1199, 2001.

[17] G. Bratt, A. L. Hammarin, M. Grandien et al., "BK virus as the cause of meningoencephalitis, retinitis and nephritis in a patient with AIDS," AIDS, vol. 13, no. 9, pp. 1071-1075, 1999.

[18] A. Vallbracht, J. Löhler, J. Gossmann et al., "Disseminated BK type polyomavirus infection in an AIDS patient associated with central nervous system disease," The American Journal of Pathology, vol. 143, no. 1, pp. 29-39, 1993.

[19] J. E. Vidal, M. C. Fink, F. Cedeno-Laurent et al., "BK virus associated meningoencephalitis in an AIDS patient treated with HAART," AIDS Research and Therapy, vol. 4, article 13, 2007.

[20] B. G. Hedquist, G. Bratt, A.-L. Hammarin et al., "Identification of BK virus in a patient with acquired immune deficiency syndrome and bilateral atypical retinitis," Ophthalmology, vol. 106, no. 1, pp. 129-132, 1999.

[21] P. L. Garavelli and R. Boldorini, "[BK virus encephalitis in an HIV-seropositive patient. Preliminary data]," Recenti Progressi in Medicina, vol. 93, no. 4, article 247, 2002.

[22] A. N. Kinnaird and G. M. Anstead, "Hemorrhagic cystitis and possible neurologic disease from BK virus infection in a patient with AIDS," Infection, vol. 38, no. 2, pp. 124-127, 2010.

[23] M. I. González-Fraile, C. Cañizo, D. Caballero et al., "Cidofovir treatment of human polyomavirus-associated acute haemorrhagic cystitis," Transplant Infectious Disease, vol. 3, no. 1, pp. 44-46, 2001.

[24] J. K. Wu and M. T. Harris, "Use of leflunomide in the treatment of polyomavirus BK-associated nephropathy," Annals of Pharmacotherapy, vol. 42, no. 11, pp. 1679-1685, 2008.

[25] A. Y. H. Leung, M. T. L. Chan, K.-Y. Yuen et al., "Ciprofloxacin decreased polyoma BK virus load in patients who underwent allogeneic hematopoietic stem cell transplantation," Clinical Infectious Diseases, vol. 40, no. 4, pp. 528-537, 2005. 


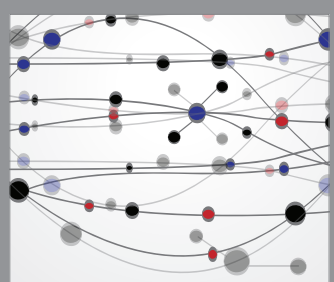

The Scientific World Journal
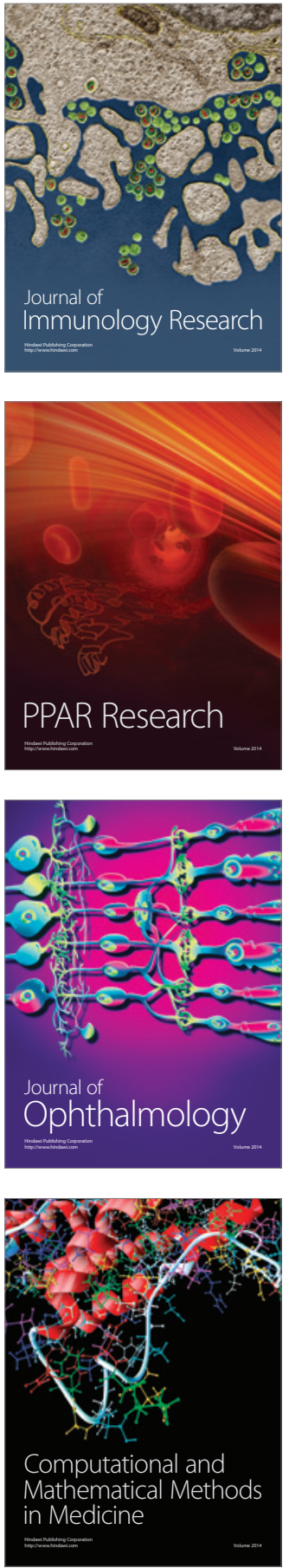

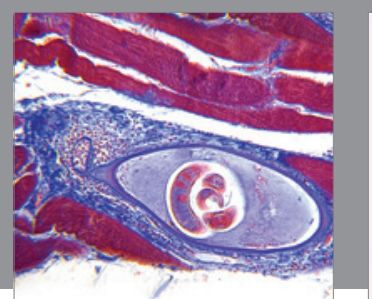

Gastroenterology Research and Practice
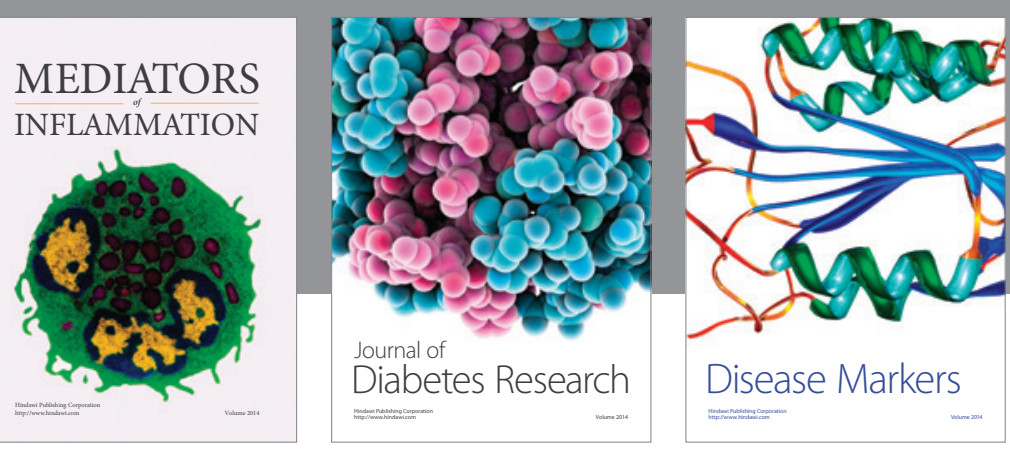

Disease Markers

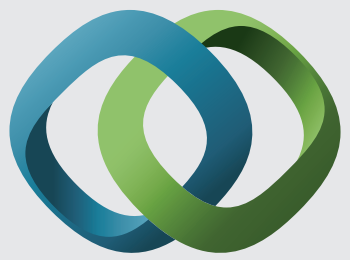

\section{Hindawi}

Submit your manuscripts at

https://www.hindawi.com
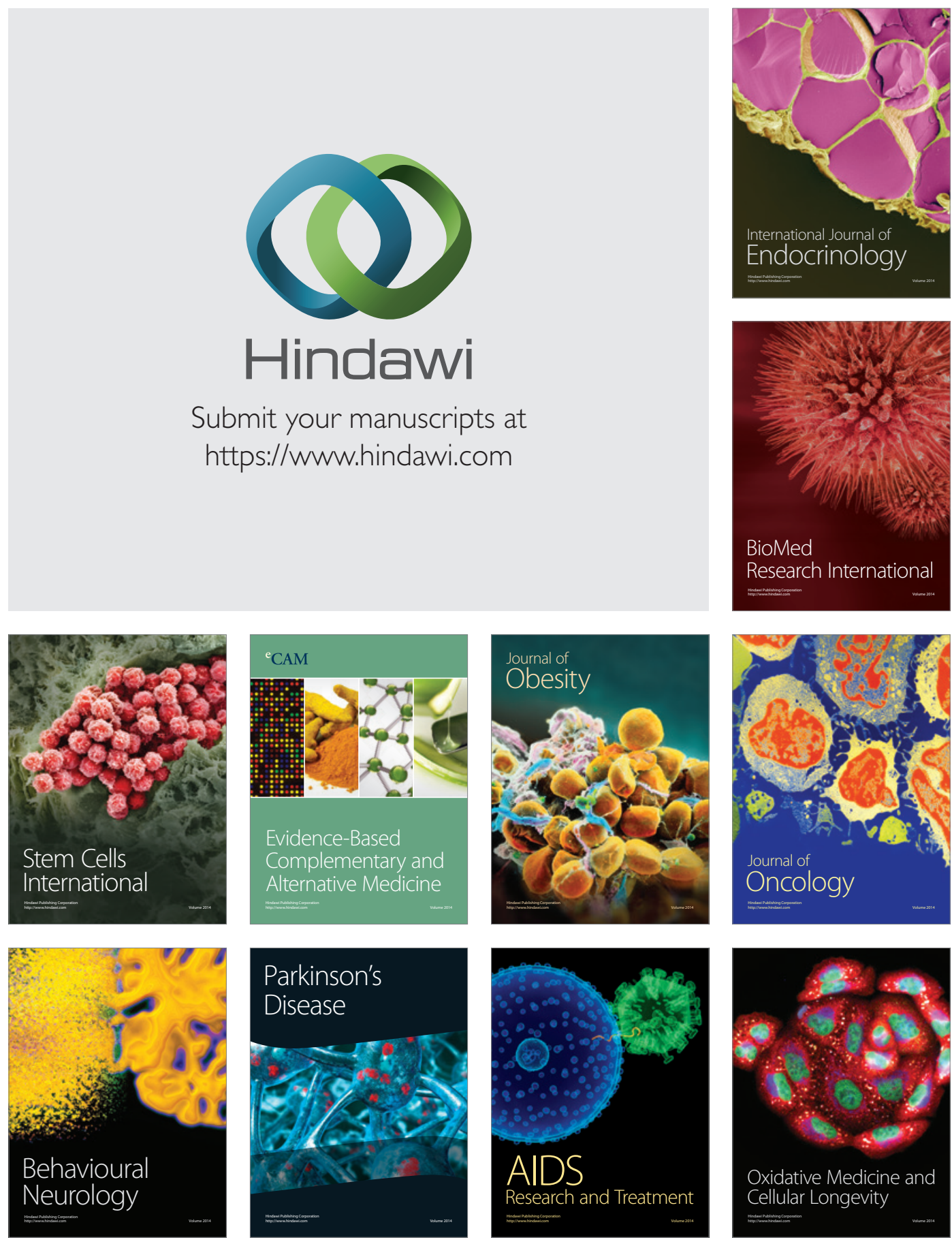